\title{
Cyanoboletus \\ macroporus (Boletaceae), \\ a new bolete species \\ from Pakistani forests
}

Samina Sarwar ${ }^{1 *}$, Arooj Naseer ${ }^{2}$ \& Abdul N. Khalid ${ }^{2}$

1 Department of Botany, Lahore College for Women University, Jail Road Campus, Lahore Pakistan

* Corresponding author:

samina_boletus@yahoo.com

Keywords: Boletaceae, Boletes, Ectomycorrhizae, Himalayan range

Article info:

Received: 07 May 2021

Accepted: 11 September 2021

Published online: 10 December 2021

Corresponding Editor: Riikka Linnakoski

\begin{abstract}
Cyanoboletus macroporus belonging to C. pulverulentus species complex is designated as a new species from the moist temperate and sub-alpine oak forests of Pakistan after in depth macroscopic, microscopic and phylogenetic analyses using the ITS region of nrDNA as well as comparison with allied taxa. This species belonging to Boletoid group is morphologically distinguished from allied taxa (Cyanoboletus flavosanguineus, C. hymenoglutinosus, C. pulverulentus, C. rainisii, and C. sinopulverulentus) by wider openings of pores. C. macroporus is also phylogenetically distinct from $C$. sinopulverulentus and $C$. pulverulentus, the most closely related spe-
\end{abstract}


cies. Phylogenetic analysis outlined the existence of previously unknown species of this genus. Field photographs of fresh basidocarps and line drawings of micro-characters are provided along with a phylogenetic tree as well as a comparison table and a key of distinctive features of all the species in this genus. This is the first authentic species belonging to Cyanoboletus from Pakistan. Previously, only $C$. pulverulentus has been mentioned in literature, but no morphological data is available regarding this report. With the addition of this taxon, species number of Cyanoboletus will increase to eight.

\section{Introduction}

Cyanoboletus is a fungal genus in the family Boletaceae circumscribed in 2014. Currently, the genus accommodates seven species: Cyanoboletusflavosanguineus (Lavorato \& Simonini) Pierotti, C. rainisii (Bessette \& O.K. Mill.) Gelardi, Vizzini \& Simonini (North America), C. sinopulverulentus (Gelardi \& Vizzini) Gelardi, Vizzini \& Simonini, C. hymenoglutinosus D. Chakr., K. Das, A. Baghela, S.K. Singh \& Dentinger, C. brunneoruber G. Wu \& Zhu L. Yang, C. instabilis (W.F. Chiu) G. Wu \& Zhu L. Yang and the type, C. pulverulentus (Opat.) Gelardi, Vizzini \& Simonini (Gelardi et al. 2013, Vizzini 2014, Pierotti 2015, Li et al. 2016, Wu et al. $2016 \mathrm{a}, \mathrm{b})$. The generic name is derived from the Ancient Greek cyano («blue»), referring to the deep ultramarine blue bruising oxidation reaction of the fruit bodies (Vizzini 2014). The type species of this genus is C. pulverulentus, commonly known as the ink stain bolete. It is an edible bolete mushroom (Smotlacha \& Vejrych 1947) growing in ectomycorrhizal association with deciduous and mixed forests, particularly on moist soil on slopes and under beech and oak trees. C. pulverulentus is a common species found in northern Asia, Europe, North Africa, Central and northern South America, and eastern North America. All parts of the mushroom will stain dark bluish-black after handling. A recent study has revealed this mushroom hyperaccumulates arsenic (with concentrations exceeding $1000 \mathrm{mg} / \mathrm{kg}$ in dry weight) and therefore its consumption should be restricted (Cocchi \& Vescovi 1996).
From Pakistan, despite of the fact that there is great diversity of mushrooms in moist temperate areas (Yousaf et al. 2012), this is the first study that describes a species belonging to Cyanoboletus genus. Previously only one ambiguous species, Cyanoboletus pulverulentus, has been mentioned in literature (Iqbal \& Khalid 1996), but with no available materials that could confirm this finding. In this study, Cyanoboletus macroporus is described as a new to science and increase the current species number of Cyanoboletus to eight.

\section{Materials and methods}

\section{Sampling and morphological analyses}

Sporocarps were collected from different localities of (moist temperate forests dominated by Quercus incana) Swat $\left(34.34-35.55^{\circ} \mathrm{N} 72.08-72.50^{\circ} \mathrm{E}\right)$, in Toa, (Shangla district), Sultanr, Khyber Pakhtunkhwa Province, Pakistan, as well as from Himalayan moist temperate forests of Pakistan dominated by conifers. Basidiocarps were photographed in the field. Fresh morphological characters were recorded in the field and colors were recorded following Munsell (1994) by Color Meter application (Yanmei He, Mac App Store). Field data on basidiocarps (site, habitat, association, etc.) was noted represented in result section below. Specimens were studied plectologically in the laboratory following the methods of Bessette et al. (2000). For the spore dimensions, the first values represent the range of lengths and widths, and the values in parentheses present mean spore lengths and widths \pm standard deviations followed $\mathrm{Qm} \pm$ standard deviation, where $\mathrm{Qm}$ is the mean of $\mathrm{Q}$ (= length/width ratio of an individual spore). Other measurements are given as a range with exceptional values in parentheses. Voucher specimens were deposited in the Herbarium, Department of Botany, University of the Punjab, Lahore, Pakistan (LAH). Voucher numbers are mentioned in holotype material and material examined section. 
DNA extraction, amplification, and sequencing

DNA was extracted from dried material of two mature sporocarps (collection numbers are mentioned in material examined and holotype) following the protocol by a modified CTAB extraction method (Gardes \& Bruns 1996). The extracted genomic DNA was evaluated using gel electrophoresis ( $1 \%$ agarose gel in Gel documentation system Uvipro Platinum (IM-2000 WL/ LC/26M MANZ) with default settings. After getting gel images, genomic DNA was suspended in nuclease free water and stored at $-18^{\circ}$ C. PCR conditions (denaturation at $94^{\circ} \mathrm{C}$ for $4 \mathrm{~min}$ followed by 35 cycles of $45 \mathrm{~s}$ at $94^{\circ} \mathrm{C}, 45 \mathrm{~s}$ at $54^{\circ} \mathrm{C}$ and $1 \mathrm{~min} 30 \mathrm{~s}$ at $72^{\circ} \mathrm{C}$, and a final extension at $72^{\circ} \mathrm{C}$ for 2 min) were used to amplify the entire rDNA internal transcribed spacer region $(\mathrm{ITS}=\mathrm{ITS} 1+5.8 \mathrm{~S}+\mathrm{ITS} 2)$ with primer pair ITS1F/ITS4 (White et al. 1990). The PCR amplicons were sent to Macrogen (Republic of Korea) for purification and bidirectional sequencing.

\section{Sequence alignment and}

phylogenetic analysis

Consensus sequences were generated from the obtained sequences with the forward and reverse algorithms in BioEdit (Hall 1999). The data was preliminary identified using BLAST searches at NCBI (http://www.ncbi.nlm.nih.gov/). The most similar sequences after BLAST in NCBI for ITS region were retrieved from GenBank. The aim was to retrieve at least 1-2 sequences of each species of Cyanoboletus genus that belongs to different countries. No sequence of this genus was present in GenBank database from Pakistan before this study. These sequences were then aligned using MUSCLE (Edgar 2004) alignment tool available online (www.ebi.ac.uklTools/msalmuscle). Maximum likelihood tree (mostly used by mycologists for phylogenetic analysis) was inferred for each of the two alignments using RAxML-HPC2 v 8.1.11 (Stamatakis 2014) with a GTR + gamma model of nucleotide substitution. One thousand bootstrap iterations were performed with rapid bootstrapping. Significant support was considered to be $\geq 70 \%$. All phylogenetic analyses were performed on the CIPRES Portal v. 3.1. (Miller et al. 2010).

\section{Results}

Molecular phylogenetic analyses

The extracted DNA from Cyanoboletus macroporus was amplified by ITS1F and ITS4 primers for ITS region. Both collections were used for molecular analysis. ITS sequences (as mentioned in Holotype and material examined) were obtained successfully and processed further for phylogenetic analysis, Consensus sequence of 678 base pairs was obtained by trimming the motifs and subjected to BLAST search at NCBI. It showed $89 \%$ identity to Boletus pulverulentus Opat. (EU819453 \& EU819502) from Western Wisconsin and $88 \%$ to Boletus sinopulverulentus (KC579402) from China with 99\% query coverage and $0.0 \mathrm{E}$ value. To analyze phylogeny, ITS sequences of different species of Cyanoboletus genus were retrieved from the GenBank and also added from literature. There is scarce data about Cyanoboletus in databank. There were 29 sequences in the final dataset (Table 2) and a total of 970 characters in the alignment file after trimming the ends at conserve sites to begin and end with the conserved motifs 5 ' -(...GAT)CATTA - and - GACCT(CAAA...)- 3 ' to facilitate alignment. These motifs correspond to the conserved 3 ' and 5 ' termini of the flanking SSU and LSU genes, respectively. Among these 236 characters were constant, 688 variable characters were parsimony-uninformative while 526 characters were parsimony informative. The sequences generated during this study get separated from closest taxa forming its own lineage in same clade with strong bootstrap value (Fig. 3).

\section{Taxonomy}

Cyanoboletus macroporus Sarwar, Naseer \&
Khalid, sp. nov. MycoBank:-MB837538

DIAGNOSIS: Cyanoboletus macroporus is characterized by dark brown pileus, Instantly basidiomata color change to olivaceous black to dark greenish black, stipe without reticulations, reddish tint near 


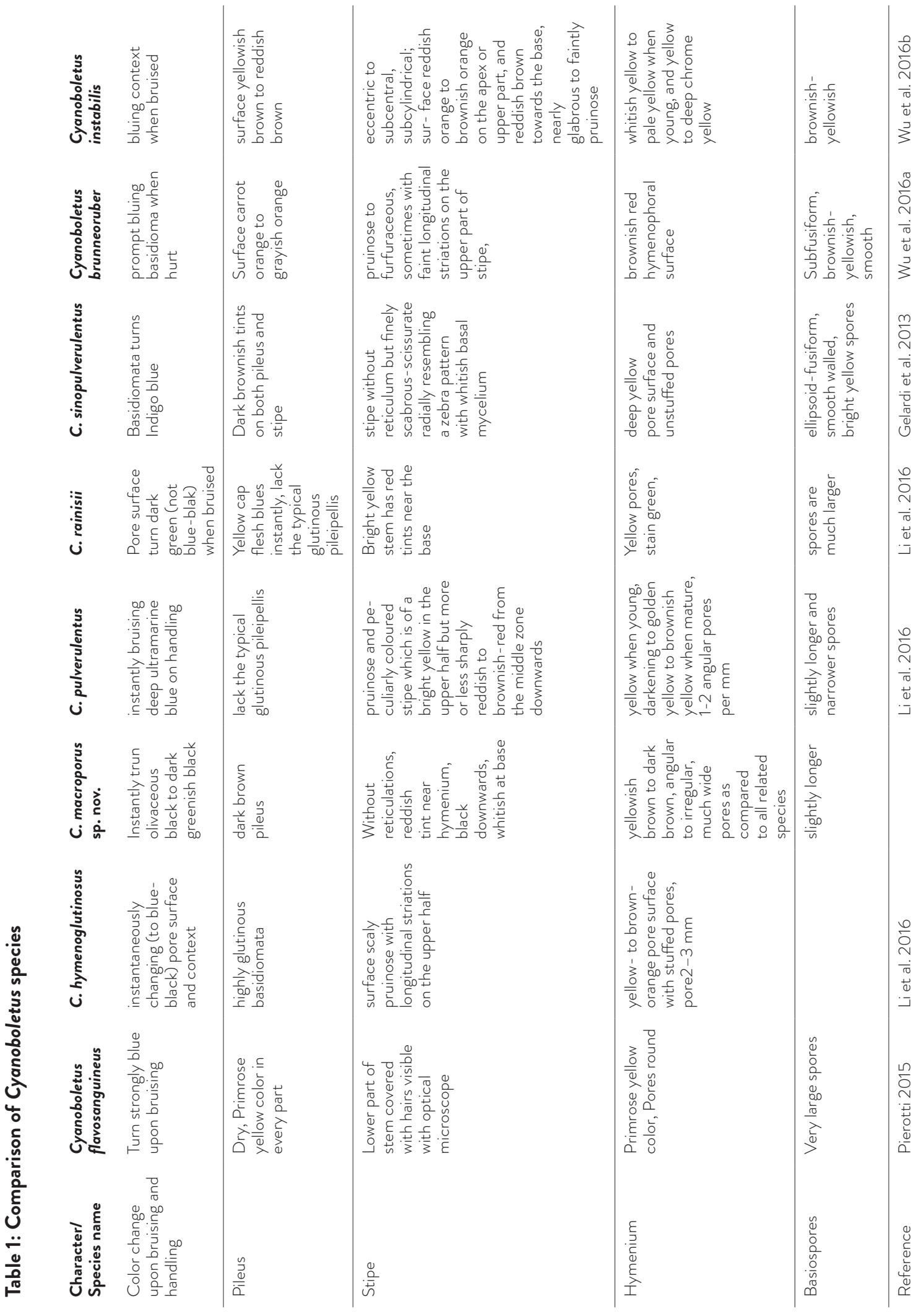




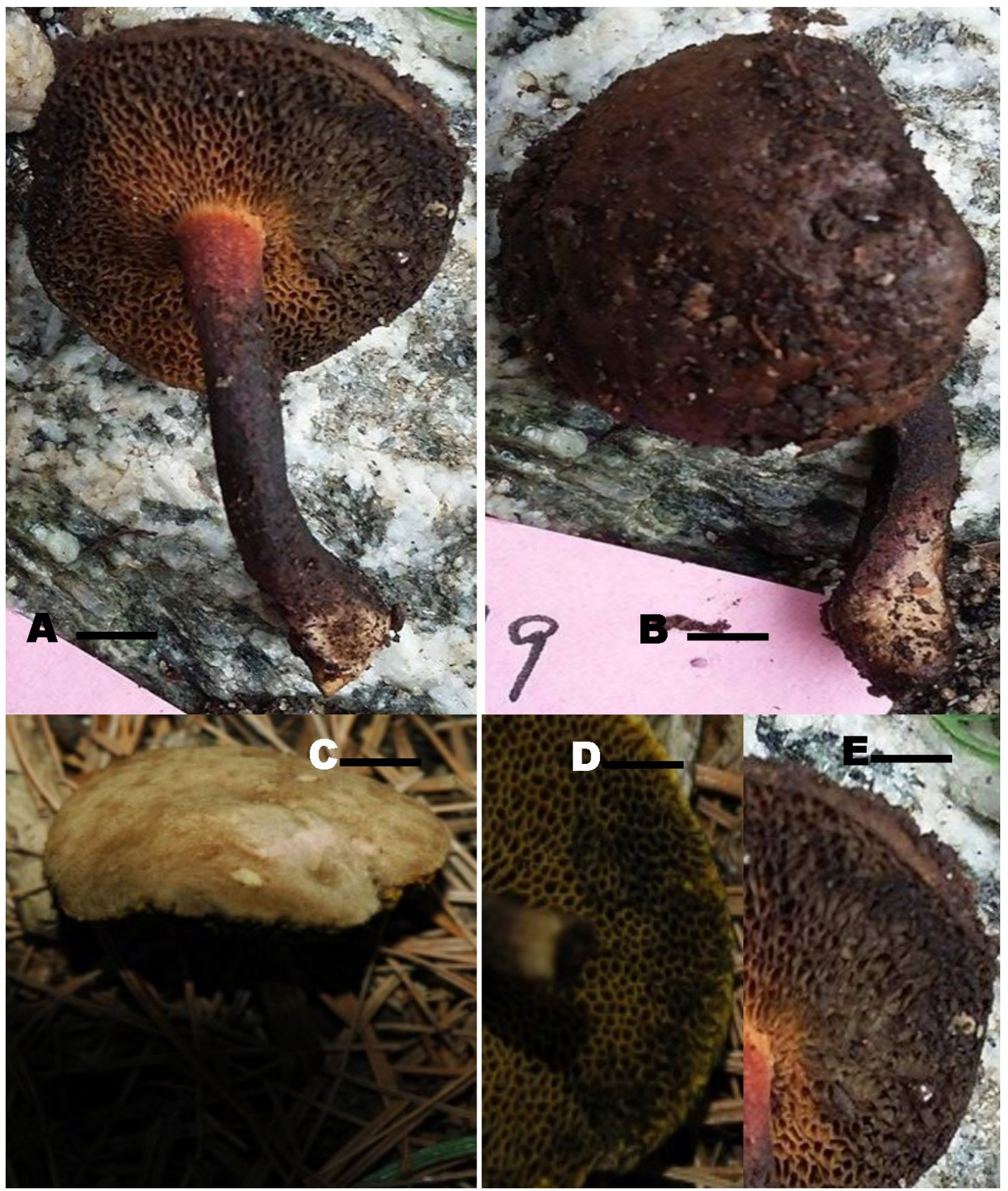

Figure 1. Morphology of Cynoboletus macroporus. A-C.

Basidioma, D-E. Pore surface. Scale Bars A-C $=0.345 \mathrm{~cm}$. 
Table 2. Information of ITS sequences retrieved for phylogenetic purpose, their sequence accession number, country of origin, host tree/habitat and reference to published work where those sequences were obtained.

\section{Taxon}

Cyanoboletus sp.

Xerocomellus rainisiae

Cyanoboletus sinopulverulentus

Boletus rainisii

Boletus rainisii

Boletus rainisii

Xerocomellus rainisiae

Rubroboletus rhodoxanthus

Boletus of subluridellus

Lanmaoa pallidorosea

Lanmaoa pallidorosea

Cyanoboletus hymenoglutinosus

Cyanoboletus sp.

Boletus pulverulentus

Boletus pulverulentus

Cyanoboletus sp.

Cyanoboletus pulverulentus

Cyanoboletus pulverulentus

Cyanoboletus pulverulentus

Cyanoboletus pulverulentus

Cyanoboletus pulverulentus

Boletus sinopulverulentus

Cyanoboletus macroporus

Cyanoboletus macroporus
ITS sequence accession number

Country

KT990767

KT990766

KU144790

MH684757

KM213664

KM213663

KM213662

KU144789

MH011921

MN128246

MN906175

MN906173

NR 164239

KT907355

EU819453

EU819502

LT714710

MT939495

LT714709

LT714708

KT157055

KT157054

KC579402

MW045557

MW369503
China

China

USA

India

USA

USA

USA

USA

France

USA

USA

USA

India

India

USA

USA

Czech Republic

USA

Czech Republic

Czech Republic

Italy

Italy

Italy

Pakistan

Pakistan reference

Wu et al. 2016b

Wu et al. 2016b

Frank et al. 2020

Chakraborty 2018

Frank et al. 2020

Frank et al. 2020

Frank et al. 2020

Unpublished

Unpublished

Unpublished

Unpublished

Unpublished

Li et al. 2016

Unpublished

Palmer et al. 2018

Palmer et al. 2018

Unpublished

Unpublished

Unpublished

Unpublished

Gelardi et al. 2015

Gelardi et al. 2015

Li et al. 2016

Present Study

Present Study 
hymenium, black downwards, whitish at base, pore surface yellowish brown to dark brown, angular to irregular, much wide pores as compared to all related species and slightly longer ellipsoid basidiospores. Phylogenetically nested in the C. pulverulentus clade.

HOLOTYPE: PAKISTAN. Khyber Pakhtunkhwa Province: Sulatanr, Swat Valley, 3400 m a.s.l., on soil, under Quercus semicarpifolia, 14 August 2015, Arooj Naseer and Abdul Nasir Khalid, ASM9 (LAH35252, GenBank MW045557).

ETYMOLOGY: The epithet 'macroporus' refers to the large size of pores in hymenium.

DESCRIPTION: Basidiomata Large, dry, change instantly olivaceous black to dark greenish black on touch. Pileus $2.5 \times 2.0 \mathrm{~cm}$ diam., broadly convex, dark brown in color (1YR 1.4/1.6), margins slightly incurved, smooth, entire. Hymenophore yellowish brown to dark brown (1YR 1.4/1.6) towards margins, angular to irregular, much wider pores. Stipe without reticulations, reddish tint near hymenium, black downwards, whitish at base, $2.8 \mathrm{~cm}$ long, $0.4 \mathrm{~cm}$ at apex, $0.2 \mathrm{~cm}$ at centre, $0.6 \mathrm{~cm}$ at base in width, dark chocolate brown (9.3RP 1.4/1.6) with brownish red (6.6R 3.5/6.6) top and grayish white (4.8YR 5.5/3.6) base, cylindrical, solid, tough, not ornamented.

Basidiospores slightly longer, 12-14 × 4-7 $\mu \mathrm{m}$, olive green in $5 \% \mathrm{KOH}$, ellipsoid, smooth, non-reticulate, guttulate sometimes bi guttulate. Basidia $23-38 \times 6-12 \mu \mathrm{m}$, olive green in $5 \% \mathrm{KOH}$, guttulate, 4 sterigmata, mostly 2, blunt ended. Pileocystidia 39$68 \times 10-12 \mu \mathrm{m}$ in diameter, light brown in $5 \% \mathrm{KOH}$, fusiform to fusi-ventricose, normal wall thickness, oil content. Caulocystidia 2-5.0 × 6-12 $\mu \mathrm{m}$ diameter, fusiform, light brown in $5 \% \mathrm{KOH}$. Pilepellis $4.0 \times$ $8.9 \mu \mathrm{m}$ in diameter, hyphal arrangement cutis, light brown in color, septate, cylindrical cell, thin walled, ends are round, yellowish brown pigments. Stipetipellis $3 \times 7 \mu \mathrm{m}$ in diameter, hyphal arrangement cutis but with broader cell, septate.

HABIT, HABITAT AND DISTRIBUTION: Swat, Sulatanr, solitary, under the canopy of Quercus semicarpifolia; Khanspur, Ayubia, Solitary, under mixed conifers.

ADDITIONAL MATERIAL EXAMINED: Khanspur, Ayubia, mixed Coniferous forests, $15^{\text {th }}$ July, 2011, S. Sarwar \& A. N. Khalid, SSB1 (LCWH12011, GenBank for ITS: MW369503).
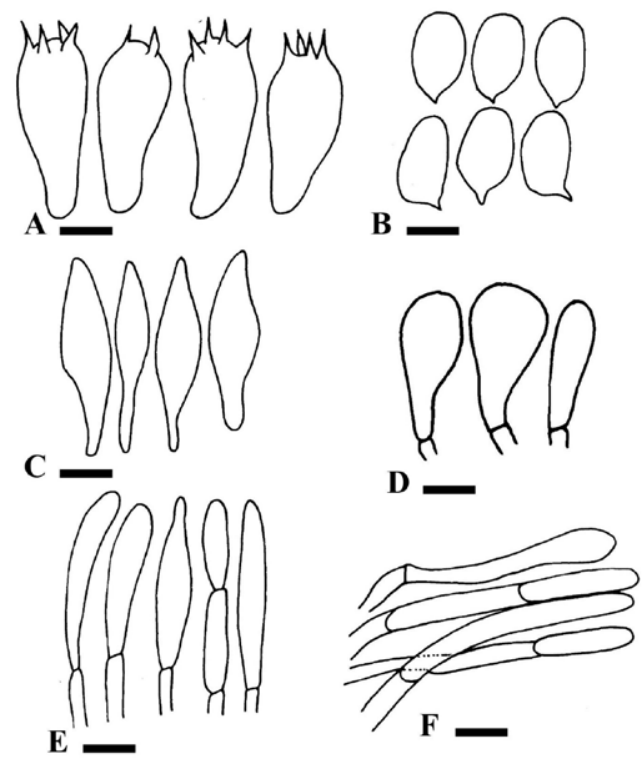

Figure 2. Anatomy of Cynoboletus macroporus. A-F LAH35252, A. Basidia; B. Basidiospores; C. Cheilocystidia; D. Pleurocystidia; E. Pileipellis; F. Stipitipellis. Scale Bars: $A=8.28 \mu \mathrm{m} ; B=5.5$ $\mu \mathrm{m} ; C$ \& $D=18 \mu \mathrm{m} ; E$ \& F = $14.25 \mu \mathrm{m}$. Illustrator \& Photographer: Dr. Arooj Naseer \& Dr. Samina Sarwar

\section{Discussion}

The genus Cyanoboletus was erected in 2014 to accommodate three existing species that were phylogenetically shown as a clade distinct from Boletus (Wu et al. 2014, Vizzini 2014). It is typified by the European Cyanoboletus pulverulentus. All three species exhibit an intense bluing colour reaction in the flesh when exposed to air, which, although not unique to the group, is a distinctive field character uniting them. They associate with both coniferous and broadleaf trees worldwide. Eight species are currently accepted in the genus (www. speciesfungorum. org). 


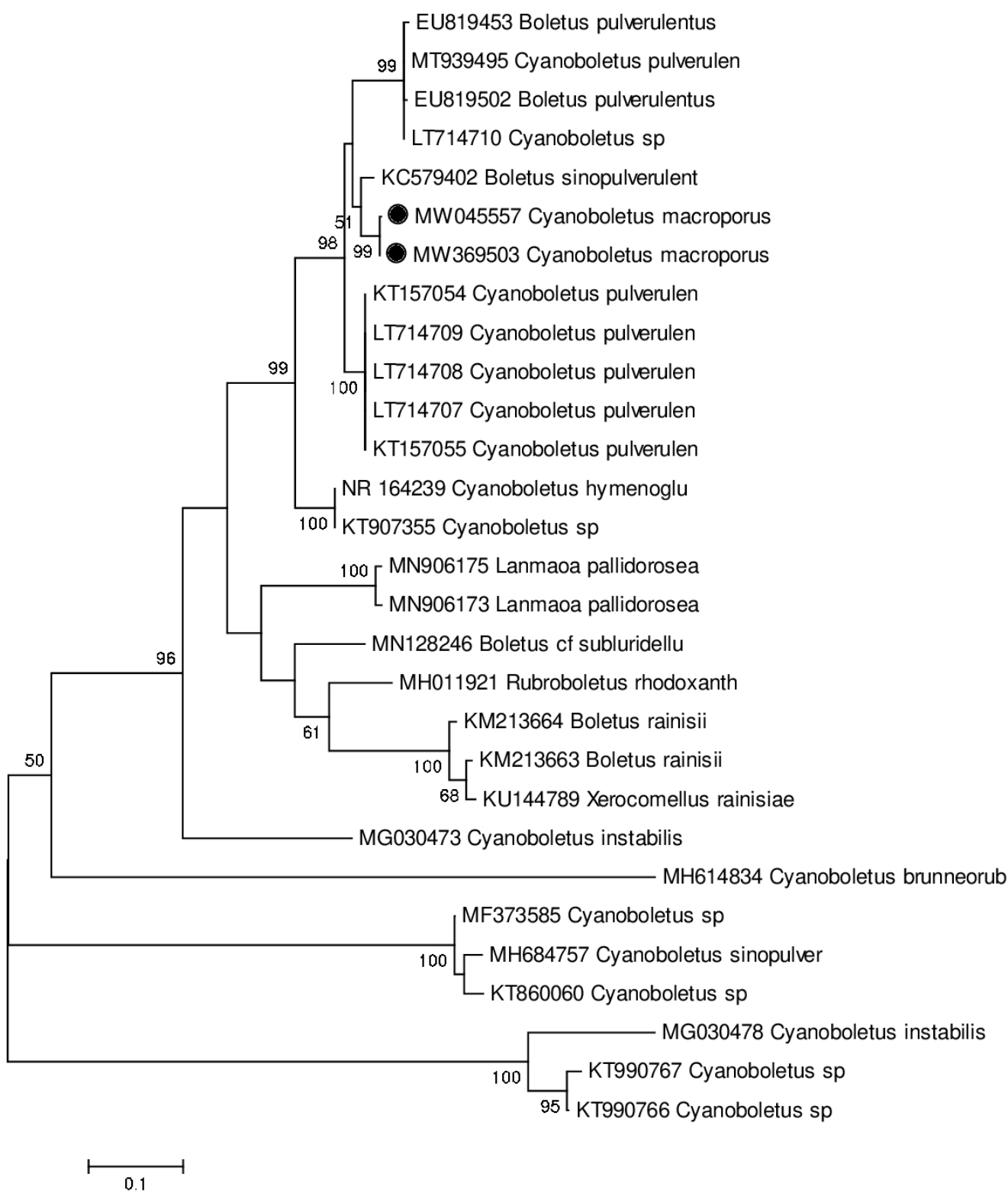

Figure 3. Phylogenetic position of Cynoboletus macroporus from Pakistan with respect to other related spp.

Tree inferred by maximum likelihood analysis based on rDNA ITS region. The numbers in branches indicate the percentage $(>50 \%)$ at which a given branch was supported in 1000 bootstrap replications. GenBank accession numbers are given at the end of species names. Highlighted taxa indicate species reported from Pakistan. Illustrator: Dr. Samina Sarwar (Corresponding Author) 
Cyanoboletus macroporus described as a novel in this study is characterized by dark brown pileus, instantly basidiomata color change to olivaceous black to dark greenish black, stipe without reticulations, pore surface with much wide pores as compared to all related species and slightly longer basidiospores. Macromorphologically, Cyanoboletus pulverulentus, C. sinopulverulentus, C. hymenoglutinosus and C. rainisii are similar to the present species.

C. macroporus differ from C. sinopulverulentus due to instantly bruising reaction that is olivaceous black to dark greenish black of basidiomata, pore surface yellowish brown to dark brown, with much wider pores in former while Indigo blue color reaction of basidiomata and deep yellow pore surface and finely scabrous-scissurate radially resembling a zebra pattern on stipe in latter. Similarly, from C. macroporus, another closely related taxa C. pulverulentus can be distinguished by instantly bruising deep ultramarine blue on handling and narrow pores. From C. hymenoglutinosus it can be differentiated due to highly glutinous basidiomata and its narrow pores. C. macroporus lack the typical glutinous pileipellis and has wider pores. All other species (Table 1) can be separated from this species due to their narrow pores that is remarkable feature of C. macroporus. C. flavosanguineus can also be differentiated due to its lower part of stem covered with hairs visible with optical microscope. C. rainisii has bright yellow glabrous stipe with reddish hues at the extreme base (Smith \& Thiers 1971, Bessette et al. 2010, Gelardi et al. 2013, Vizzini 2014).

Molecular and phylogenetic analysis also supports the morphological comparisons. C. macroporus clusters as an independent species, as sister to $C$. sinopulverulentus, they are in turn sister to C. pulverulentus sequences. Sequences of $C$. macroporusfalls in C. pulverulentus clade but showed its distinct identity from C. sinopulverulentus and C. pulverulentus (Fig. 3). Both morphological and molecular analysis showed that $C$. macroporus is a new species.

\section{Key to Cyanoboletus species}

\author{
Key distinguishing feature of genus
}

Deep ultramarine blue bruising reaction of the fruit bodies. Cyanoboletus

Lower part of stem covered with hairs visible with optical microscope......C. flavosanguineus

highly glutinous basidiomata, instantaneously changing (to blue-black) pore surface and context.......C. hymenoglutinosus

wider pores....C. macroporus sp. nov.

young cap and stipe covered with powder..... C. pulverulentus.

bright yellow glabrous stipe with reddish hues at the extreme base, instantly stain greenish-black, ...... r. rainisii

stipe without reticulum but finely scabrous-scissurate radially resembling a zebra pattern and yellow pore surface....... sinopulverulentus

\section{Acknowledgments}

Authors are grateful to Higher Education Commission of Pakistan for providing financial support for this research work.

\section{Conflict of Interest}

This work is original and there is no conflict of interest.

\section{References}

Bessette, A.E., Roody, W.C. \& Bessette, A.R. (2000) North American boletes: a color guide to the fleshy pored mushrooms, Syracuse University Press, Syracuse, New York. 396 p.

Cocchi, L. \& Vescovi, L. (1996) Considerazioni sulle concentrazioni di elementi chimici in funghi dell'Ordine Boletales. II Fungo, Associazione Micologica Bresadola. 42-60. 
Chakraborty, D., Vizzini, A. \& Das, K. (2018) Two new species and one new record of the genus Tylopilus (Boletaceae) from Indian Himalaya with morphological details and phylogenetic estimations. MycoKeys 33: 103-124.

Edgar, R.C. (2004) MUSCLE: multiple sequence alignment with high accuracy and high throughput. Nucleic Acids Research 32: 1792-1797.

Frank, J.L., Siegel, N., Schwarz, C.F., Araki, B. \& Vellinga, E.C. (2020) Xerocomellus (Boletaceae) in western North America. Fungal Systematics and Evolution 6: 265-288. doi: 10.3114/fuse.2020.06.13

Gardes, M. \& Bruns, T.D. (1996) ITS-RFLP matching for identification of fungi. In: Clapp JP, ed. Methods in molecular biology, Vol. 50. Species diagnostics protocols: PCR and other nucleic acid methods. Totowa, NJ, USA: Humana Press Inc., 177-186.

Gelardi, M., Vizzini, A., Ercole, E., Voyron, S., Sun, J.Z. \& Liu, X.Z. (2013) Boletus sinopulverulentus, a new species from Shaanxi Province (central China) and notes on Boletus and Xerocomus. Sydowia 65(1): 45-57.

Gelardi, M., Simonini, G., Ercole, E., Davoli, P. \& Vizzini, A. (2015) Cupreoboletus (Boletaceae, Boletineae), a new monotypic genus segregated from Boletus sect. Luridi to reassign the Mediterranean species $B$. poikilochromus. Mycologia 107: 1254 - 1269. https://doi. org/10.3852/15-070

Hall, T. (1999) BioEdit: a user-friendly biological sequence alignment editor and analysis program for windows 95/98/ NT. Nucleic Acids Symp. Ser. 41: 95-98.

Iqbal, S.H., Khalid, A.N. (1996) Material for the fungus flora of Pakistan. I. Check list of Agarics, their distribution and association with the surrounding vegetation.

Science International (Lahore) 8: 51-64.

Li, G.J., Hyde, K.D. \& Zhao, R.L., et al. (2016) Fungal diversity notes 253-366: taxonomic and phylogenetic contributions to 3 fungal taxa. Fungal Diversity 78(1).

https://doi:10.1007/s13225-016-0366-9

Miller, M.A., Pfeiffer, W. \& Schwartz, T. (2010) Creating the CIPRES Science Gateway for inference of large phylogenetic trees. Gateway Computing Environments Workshop (GCE). IEEE, New Orleans, LA, 1-8.

https://doi.org/10.1109/GCE.2010.5676129

Munsell Soil Colour Charts. (1994) Macbeth, New Windsor.
Palmer, J., Lindner, D. \& Volk. T. (2008) Ectomycorrhizal characterization of an American chestnut (Castanea dentata)-dominated community in western Wisconsin. Mycorrhiza 19: 27-36.

Pierotti, A. (10 October 2015) "Nomenclatural novelties". Index Fungorum 263.

Smith, A.H. \& Thiers, H.D. (1971) The Boletes of Michigan. Ann Arbor: Univ. Michigan Press. 428 p.

Smotlacha, F. \& Vejrych, R. (1947) 50 recommended edible mushrooms (In Czech). Prague: Unie130.

Stamatakis, A. (2014) RAxML version 8: a tool for phylogenetic analysis and post-analysis of large phylogenies. Bioinformatics 30: 1312-1313.

Vizzini, A. (7 June 2014) "Nomenclatural novelties". Index Fungorum 176: 1.

White, T.J., Bruns, T., Lee, S. \& Taylor, J. (1990) Amplification and direct sequencing of fungal ribosomal RNA genes for phylogenetics. In: Innis MA, Gelfand DH, Sninsky JJ, White TJ, eds. PCR Protocols: a guide to methods and applications. San Diego, CA, USA: Academic Press, Inc., 315-322.

Wu, G., Feng, B., Xu, J., Zhu, X.T., Li, Y.C., Zeng, N.K., Hosen, M.I. \& Yang, Z.L. (2014) Molecular phylogenetic analyses redefine seven major clades and reveal 22 new generic clades in the fungal family Boletaceae. Fungal Diversity 69: 93-115. https://doi.org/10.1007/s13225-014-0283-8

Wu, G., Li, Y.C., Zhu, X.T., Zhao, K., Han, L.H., Cui, Y.Y., Li, F., Xu, J.P. \& Yang, Z.L. (2016b) One hundred noteworthy boletes from China. Fungal Diversity 81: 25-188. https://doi:10.1007/s13225-016-0375-8

Wu, G., Zhao, K., Li, Y.C., Zeng, N.K., Feng, B., Halling, R. \& Yang, Z.L. (2016a) Four new genera of the fungal family Boletaceae. Fungal Diversity. https://doi:10.1007/s13225-015-0322-0

Yousaf, N., Niazi, A.R., Khalid, A.N. (2012) New records of noteworthy gasteroid fungi from Pakistan. Mycotaxon 119: 261-267. doi:10.5248/119.261 